\title{
Comment on: MicroRNA Expression as a Predictive Marker for Gemcitabine Response After Surgical Resection of Pancreatic Cancer
}

\author{
Sameer A. Dhayat, MD, Wolf Arif Mardin, MD, and Norbert Senninger, MD, FACS \\ Department of General and Visceral Surgery, University Hospital of Muenster, Muenster, Germany
}

\section{TO THE EDITORS:}

We read with great interest the study by Ohuchida et al. investigating microRNAs (miRNAs) as prognostic biomarkers for chemoresistance in pancreatic ductal adenocarcinoma (PDAC). ${ }^{1}$ We congratulate the authors for advancing the research on molecular markers predicting chemotherapy response in this aggressive carcinoma entity. Their translational study evaluates in vitro results in a clinical setting and is an important contribution towards understanding and overcoming chemoresistance in PDAC patients. Deregulated miRNAs were retrospectively investigated in patients who underwent resection for PDAC. Expression profiling identified several miRNAs aberrantly expressed in two human PDAC cell lines and clinical tissue samples that were significantly correlated with clinical outcome, most notably miRNA-142-5p, which was an independent prognostic marker for longer survival in patients treated with gemcitabine.

These findings are highly interesting and the identified miRNAs may prove useful in the prediction of the individual chemotherapy response, and even provide therapeutic targets for increasing chemosensitivity in PDAC patients.

However, issues that deserve further clarification remain. It is necessary to know the exact number of included patients with adjuvant gemcitabine monotherapy, and the patients' respective Union for International Cancer Control (UICC) stages and their assignment to adjuvant or palliative treatment groups is important. A subgroup

(C) Society of Surgical Oncology 2017

First Received: 20 October 2017;

Published Online: 1 November 2017

S. A. Dhayat, MD

e-mail: sameer.dhayat@ukmuenster.de analysis of these powerful predictors that could have distorted the present results would rule out such a bias and greatly enhance the present results.

Contamination by surrounding stroma and/or inflammatory tissue is unavoidable when using macrodissected cancer tissue, thus masking the miRNA expression profile of the neoplastic cells. Techniques such as laser microdissection can help minimize such contaminations by isolating cancer cells from specimens in a manner that allows a more precise extraction of miRNAs. ${ }^{2}$

Interpretation of the prognostic value of single miRNAs in clinical samples is based on in vitro expression profiling of chemoresistant cell lines. We would be grateful for further information about the number and duration of therapy cycles until chemoresistant cell lines were established and if gemcitabine was applied in a pulsatile mode or continuously. These are methodological details that are essential if the present results are to be compared with other studies.

Overall, the results of the study by Ohuchida et al. are highly encouraging and we concur that further validation of the prognostic value of miRNA-142-5p is warranted. Prospective studies with greater numbers of well-characterized PDAC patients will enhance our understanding of the mechanisms underlying chemoresistance and increase our knowledge of the complex interactions between the implicated miRNAs and chemotherapeutic agents.

\section{REFERENCES}

1. Ohuchida K, Mizumoto K, Kayashima T, et al. MicroRNA expression as a predictive marker for gemcitabine response after surgical resection of pancreatic cancer. Ann Surg Oncol. 2011;18(8):2381-87.

2. Giovannetti E, Funel N, Peters GJ, et al. MicroRNA-21 in pancreatic cancer: correlation with clinical outcome and pharmacologic aspects underlying its role in the modulation of gemcitabine activity. Cancer Res. 2010;70(11):4528-38. 